\title{
Addition of Vardenafil into Storage Solution Protects the Endothelium in a Hypoxia-Reoxygenation Model
}

\author{
G. Veres $^{\text {a,b, }{ }^{*}, d}$, P. Hegedüs ${ }^{\text {a,c,d }}$, E. Barnucz ${ }^{a, c}$, R. Zöller ${ }^{\text {a }}$, T. Radovits ${ }^{\text {a,c }}$, S. Korkmaz ${ }^{\text {, }}$ F. Kolonics ${ }^{\text {a,c }}$, A. Weymann ${ }^{\text {a }}$, \\ M. Karck a , G. Szabó a \\ ${ }^{a}$ Department of Cardiac Surgery, University of Heidelberg, Germany \\ ${ }^{b}$ Department of Cardiac Surgery, Semmelweis University, Hungary \\ ${ }^{\mathrm{c}}$ Heart Center, Semmelweis University, Hungary
}

WHAT THIS PAPER ADDS

During bypass surgery, preservation of vascular grafts in preservation solutions (saline or Custodiol) causes endothelial dysfunction, leading to an enhanced rate of complications and unfavourable early and late outcome (e.g., early graft thrombosis, vasospasm, restenosis, and occlusion). Additionally, there are unavoidable situations in vascular or transplantation surgery in which a long ischaemic period is necessary and therefore current storage protocols need further improvement. We proved in our model that vardenafil-enriched solution efficiently preserved the endothelium after long-term cold ischaemic storage and warm reperfusion, and could be a new therapeutic option for an optimal preservation of vascular grafts.

Objective: Based upon the well known protective effect of intracellular cyclic guanosine monophosphate (cGMP) accumulation, we tested the hypothesis that storage solution enriched with optimal concentration of the phosphodiestherase- 5 inhibitor vardenafil could provide better protection of vascular grafts against reperfusion injury after long-term cold ischaemic storage.

Methods: Isolated thoracic aorta obtained from rats underwent 24-h cold ischaemic preservation in physiological saline or vardenafil $\left(10^{-11} \mathrm{M}\right)$-supplemented saline solution. Reperfusion injury was simulated by hypochlorite $(200 \mu \mathrm{M})$ exposure for 30 minutes. Endothelium-dependent vasorelaxation was assessed, and histopathological and molecular-biological examination of the aortic tissue were performed.

Results: Compared with the control group, the saline group showed significantly attenuated endotheliumdependent maximal relaxation $\left(R_{\max }\right)$ to acetylcholine after hypoxia-reoxygenation, which was significantly improved by vardenafil supplementation ( $R_{\max }$ control: $98 \pm 1 \%$; saline: $48 \pm 6 \%$; vardenafil: $75 \pm 4 \% ; p<.05$ ). Vardenafil treatment significantly reduced DNA strand breaks (control: $10.6 \pm 6.2 \%$; saline: $72.5 \pm 4.0 \%$; vardenafil: $14.2 \pm 5.2 \% ; p<.05$ ) and increased cGMP score in the aortic wall (control: $8.2 \pm 0.6$; saline: $4.5 \pm 0.3$; vardenafil: $6.7 \pm 0.6 ; p<.05)$.

Conclusions: Our results support the view that impairment of intracellular cGMP signalling plays a role in the pathogenesis of the endothelial dysfunction induced by cold storage warm reperfusion, which can be effectively reversed by pharmacological phosphodiesterase- 5 inhibition.

(C) 2013 European Society for Vascular Surgery. Published by Elsevier Ltd. All rights reserved.

Article history: Received 13 December 2012, Accepted 8 May 2013, Available online 7 June 2013

Keywords: cGMP, Endothelium, Ischaemia-reperfusion injury, Storage, Vardenafil

\section{INTRODUCTION}

Prevention of ischaemia and reperfusion (I/R) injury in cardiac surgery is an important issue in the field of tissue and organ protection, as $\mathrm{I} / \mathrm{R}$ is a determining factor of acute and chronic graft failure. ${ }^{1}$ Just as for other organs, cold

\footnotetext{
${ }^{\mathrm{d}}$ These authors contributed equally to this work.

* Corresponding author. G. Veres, Laboratory of Cardiac Surgery, Department of Cardiac Surgery, University of Heidelberg, INF 326, 69120 Heidelberg, Germany.

E-mail address: gaborveres@yahoo.com (G. Veres).

1078-5884/\$ - see front matter (C) 2013 European Society for Vascular Surgery. Published by Elsevier Ltd. All rights reserved. http://dx.doi.org/10.1016/j.ejvs.2013.05.006
}

ischaemic storage conservation solution is preferable to avoid the loss of functional integrity of vascular grafts. Regarding the importance of graft patency, several different storage solutions for intraoperative short-time conservation of free vascular grafts have been tested in the past. Grafts are likely to be stored between $4{ }^{\circ} \mathrm{C}$ and room temperature in heparinised blood, saline and, rarely, in histidinetriptophane-ketoglutarate (HTK or Custodiol) or University of Wisconsin solutions. The basic concept behind the usual cold ischaemic storage is the suppression of metabolic activity. The deleterious side of this preservation method is cell swelling, acidosis and release of reactive oxygen species (ROS). ${ }^{2}$ Although some studies have indicated the superiority of HTK solution compared with Ringer and saline 
solutions for storing saphenous vein grafts, ${ }^{3,4}$ the poor longterm preservation capacity of HTK solution has been demonstrated by an in vitro coronary artery model. ${ }^{5}$ However, saline or HTK solutions are spread in clinical routine.

In vascular grafts during reperfusion injury due to myeloperoxidase released from activated leucocytes, the production of ROS and other related oxidants reaches pathological levels. ${ }^{6}$ ROS-like hypochlorous acid and peroxynitrite attack various biomolecules in the vasculature, causing DNA strand breakage, mitochondrial function disruption, lipid peroxidation induction, and depletion of antioxidant reserves. ${ }^{7}$ First and foremost, the enhanced formation of ROS originating from the leucocyte-endothelial cell interactions affects the endothelium, resulting in endothelial and, consequently, vascular dysfunction. ${ }^{8}$ Such dysfunction manifests in the impaired production of the thrombocyte activation inhibitor prostacycline, and in the decreased ability of vasodilatation caused by the inhibition of the nitric oxide (NO)-cyclic guanosine monophosphate (cGMP)-cGMP-dependent protein kinase (PKG) pathway. This pathway is shown to have a considerable role in vascular- and cardioprotection. ${ }^{9,10}$ Intracellular cGMP accumulation is proven to reduce tissue injury in conditions associated with increased free radical release and oxidative stress. ${ }^{11,12}$

Vardenafil is a selective phosphodiesterase (PDE)-5 inhibitor that hinders the degradation of cGMP by inhibiting its predominant regulator enzyme. Vardenafil was shown to have beneficial effects against myocardial $I / R$ injury after preconditioning-like treatment in rabbits ${ }^{13}$ and an advantageous protective effect on vascular endothelium. ${ }^{1,14}$ The protective effect of PDE-5 inhibition on endothelial dysfunction following I/R injury was also demonstrated in a human study, although sildenafil was used. ${ }^{15}$

Based upon the well known protective effect of intracellular CGMP accumulation, this study aimed to test the hypothesis that storage solution enriched with an optimal concentration of vardenafil could provide better protection of vascular grafts against reperfusion injury after long-term cold ischaemic storage.

\section{METHODS}

Please see the Supplementary material for further details on the quantification of terminal deoxynucleotidyl transferase-mediated dUTP nick-end labelling (TUNEL) and cGMP stainings, evaluation of quantitative real-time polymerase chain reaction (qRT-PCR), and Western blotting.

\section{Animals}

Male Sprague-Dawley rats (250-330 g; Charles River, Sulzfeld, Germany) were housed in a room at a constant temperature of $22 \pm 2{ }^{\circ} \mathrm{C}$ with 12 -hour light/dark cycles, and were fed a standard laboratory rat diet; water was available ad libitum. All procedures concerning animals conformed with the Guide for the Care and Use of Laboratory Animals prepared by the Institute of Laboratory Animal Resources and published by the National Institutes of Health (NIH Publication No. 86-23, revised 1996). The investigations were reviewed and approved by the local ethics committee for animal experimentation.

Experimental groups and treatment. Rats were euthanised by overdose of sodium pentobarbital before exsanguination. The left chamber was pierced with a $4 \mathrm{G}$ needle, and $20 \mathrm{~mL}$ conservation solution was infused at a speed of $1 \mathrm{~mL} /$ second transcardially to wash out any blood from the aorta. According to the experimental groups, this conservation solution was cold Krebs-Henseleit solution $(118 \mathrm{mmol} / \mathrm{L}$ sodium chloride, $4.7 \mathrm{mmol} / \mathrm{L}$ potassium chloride $[\mathrm{KCl}], 1.2 \mathrm{mmol} / \mathrm{L}$ potassium hydrogen phosphate, $1.2 \mathrm{mmol} / \mathrm{L}$ magnesium sulphate, $1.77 \mathrm{mmol} / \mathrm{L}$ calcium chloride, $25 \mathrm{mmol} / \mathrm{L}$ sodium bicarbonate, and $11.4 \mathrm{mmol} / \mathrm{L}$ glucose; $\mathrm{pH}=7.4$ ), saline, or vardenafil solution $\left(10^{-11} \mathrm{M}\right)$. Thoracic aorta was excised carefully, cleaned from connective tissue, and cut transversely into 4-mm-wide rings under an operation microscope. Special attention was paid to avoid damaging the endothelium. Aortic rings were stored in $4{ }^{\circ} \mathrm{C}$ for 24 hours in $5 \mathrm{~mL}$ cold saline or $10^{-11} \mathrm{M}$ vardenafil-containing tubes. The tubes were previously equilibrated for 15 minutes with NO to extrude oxygen from the solution. Aortic rings in the saline group received the same treatment, but with only saline transcardial perfusion and no vardenafil in the storage saline solution. After 24 hours of ischaemic storage, rings proceeded to the organ bath. To stimulate free radical burst, which occurs usually in vivo during reperfusion, ${ }^{16} 200 \mu \mathrm{M}$ hypochlorite ( $\mathrm{HOCl})$ was added for 30 minutes to the baths of the saline and vardenafil groups. Aortic rings in the control group did not go through cold ischaemic storage, but were mounted in an organ bath and received no $\mathrm{HOCl}$ exposition.

In vitro organ bath experiments. Isolated aortic rings were mounted on stainless steel hooks in individual organ baths (Radnoti Glass Technology, Monrovia, CA, USA), containing $25 \mathrm{~mL}$ of Krebs-Henseleit solution at $37{ }^{\circ} \mathrm{C}$ and aerated with $95 \% \mathrm{O}_{2}$ and $5 \% \mathrm{CO}_{2}$. Isometric contractions were recorded using isometric force transducers of a myograph (159901A; Radnoti Glass Technology), digitised, stored, and displayed with the IOX Software System (EMKA Technologies, Paris, France). The aortic rings $(n=15-20$ from $N=8-12$ animals in each group) were placed under a resting tension of $2 \mathrm{~g}$ (which was found to be optimal in preliminary experiments, ${ }^{7,17}$ ) and equilibrated for $60 \mathrm{mi}-$ nutes. During this period, tension was adjusted periodically to the desired level and the Krebs-Henseleit solution was changed every 30 minutes. At the beginning of each experiment, maximal contraction forces to $\mathrm{KCl}(80 \mathrm{mM})$ were determined and aortic rings were washed until the resting tension was again obtained. Afterwards, $200 \mu \mathrm{M}$ $\mathrm{HOCl}$ was added (except the control group) for 30 minutes to the baths, then washed out. Aortic preparations were pre-constricted with $\alpha$-adrenergic receptor agonist phenylephrine $\left(10^{-6} \mathrm{M}\right)$ until a stable plateau was reached, and relaxation responses were examined by adding cumulative concentrations of endothelium-dependent dilator acetylcholine $\left(10^{-9}\right.$ to $\left.10^{-4} \mathrm{M}\right)$. For testing the relaxation response of smooth muscle cells, a direct NO donor, sodium nitroprusside $\left(10^{-10}\right.$ to $\left.10^{-5} \mathrm{M}\right)$, was used. Half-maximal 
response $\left(E_{50}\right)$ values were obtained from individual concentration-response curves by fitting experimental data to a sigmoidal equation using Origin 7.0 (Microcal Software, Northampton, MA, USA). Contractile responses to phenylephrine are expressed as the percent of the maximal contraction induced by $\mathrm{KCl}$. The sensitivity to vasorelaxants was assessed by $\mathrm{pD}_{2}=-\log \mathrm{EC}_{50}(\mathrm{M})$; vasorelaxation (and its maximum $\left.\left(R_{\max }\right)\right)$ is expressed as the percent of contraction induced by phenylephrine $\left(10^{-6} \mathrm{M}\right)$.

Histopathological processing. Aortic segments from each experimental group were fixed in paraformaldehyde solution (4\%) and embedded in paraffin. The $3-\mu \mathrm{m}$-thick sections were placed on adhesive slides.

\section{TUNEL reaction}

Following the manufacturer's protocol (Chemicon International, Temecula, CA, USA), TUNEL staining was performed as described previously. ${ }^{17}$

cGMP immunohistochemical staining. Rehydrated sections were blocked (3\% goat serum) and rabbit polyclonal anticGMP primary antibody (Abcam, Cambridge, UK) incubation was performed $(1: 1,000)$ for 2 hours at room temperature. Tissue sections were incubated overnight at $4{ }^{\circ} \mathrm{C}^{18}$ followed by incubation of secondary biotinylated antirabbit immunoglobulin E (BioGenex, Fremont, CA, USA), which allowed the alkaline phosphatase-conjugated streptavidin to react (BioGenex). A red reaction product at the site of the target antigen was formed by Fast Red substrate (DakoCytomation, Hamburg, Germany). Negative controls were counterstained with Gill's haematoxylin.

qRT-PCR. Total RNA was isolated from the chosen aortic rings with the RNeasy Fibrous Tissue Mini Kit (Qiagen, Hilden, Germany) after homogenisation. The concentration and purity of RNA were determined at 260, 280, and $230 \mathrm{~nm}$ with a spectrophotometer. Reverse transcription was performed with the QuantiTect Reverse Transcription Kit (Qiagen) using $400 \mu \mathrm{g}$ RNA in a volume of $20 \mu \mathrm{L}$. qRTPCR was performed in the LightCycler480 system with Universal Probelibrary probes (Roche, Mannheim, Germany). Primers were obtained from TIB Molbiol (Berlin, Germany) (see Supplementary Table 1).

\section{Western blotting}

Proteins were extracted from tissue homogenates, and Western blotting was performed for the quantification of p17 caspase-3 fragment $(1: 1,000)$, Bax (1:200), and Bcl-2 (1:100; Abcam). Glyceraldehyde-3-phosphate dehydrogenase was determined as housekeeping protein (1:500; Santa Cruz Biotechnology, Heidelberg, Germany). Target protein densities were normalised to housekeeping densities of the same sample.

\section{Statistical analysis}

Data were tested for normal distribution (Shapiro-Wilk) and where they met the requirements for parametric analysis, means were tested by analysis of variance and Bonferroni's correction test. For the analysis of PCR results the Kruskal-Wallis test for multiple comparison was used. A $p$-value $<.05$ was considered statistically significant.

\section{RESULTS}

\section{Endothelium-dependent and endothelium independent vasorelaxation of aortic rings}

In aortic rings pre-contracted with phenylephrine, acetylcholine induced concentration-dependent relaxation. Aortic segments exposed to cold ischaemic storage followed by $\mathrm{HOCl}$ incubation showed significantly attenuated $R_{\max }$ to acetylcholine and significant increase of $\mathrm{pD}_{2}$, which implies a shift of the acetylcholine concentration-response curves to the right compared with the control group (Table 1, Fig. 1A). Conservation of aortic segments in vardenafil-supplemented saline solution significantly improved the acetylcholine-induced endothelium-dependent NO-mediated vasorelaxation after the exposure of rings to $\mathrm{HOCl}$ (Table 1, Fig. 1A). There was no significant different in $R_{\max }$ for endothelium-independent vasorelaxation of the aortic rings to sodium nitroprusside between the experimental groups. (Table 1, Fig. 1B).

\section{Contractile responses of aortic rings}

Contractile responses of aortic rings to phenylephrine $\left(10^{-6} \mathrm{M}\right)$ are shown in Table 1 . Incubation of aortic segments of saline and vardenafil groups with $\mathrm{HOCl}$ significantly increased phenylephrine-induced maximum contraction compared with the control group $(78.1 \pm 2.5 \%$ vs. $123.5 \pm 1.6 \%$ vs. $123.6 \pm 3.5 \%, p<.05$ control, saline, and vardenafil groups respectively). However, contractile responses to high $\mathrm{KCl}$-induced depolarisation did not differ significantly between the experimental groups (Table 1).

\section{Vardenafil decreases DNA strand breaks and maintains intracellular cGMP level}

An increased density of TUNEL-positive nuclei indicates DNA fragmentation in the aortic wall (intima and media) of rings

Table 1. Values of maximal relaxation $\left(R_{\mathrm{max}} \%\right)$ and $\mathrm{pD}_{2}$ to acetylcholine to sodium nitroprusside (SNP), and contraction forces induced by phenylephrine $\left(10^{-6} \mathrm{M}\right)$ in control, saline, or vardenafil $\left(10^{-11} \mathrm{M}\right)$ conserved hypochlorite-exposed thoracic aortic rings. Values represent mean \pm standard error of the mean.

\begin{tabular}{lrrr} 
& $\begin{array}{c}\text { Control } \\
R_{\max }\end{array}$ & \multicolumn{1}{c}{$\begin{array}{c}\text { Saline } \\
\text { to acetylcholine }\end{array}$} & \multicolumn{1}{c}{ Vardenafil } \\
(\%) & & & \\
& & & \\
$\mathrm{pD}_{2}$ & $7.6 \pm 0.1$ & $6.4 \pm 0.1^{\mathrm{a}}$ & $6.9 \pm 0.08^{\mathrm{a}, \mathrm{b}}$ \\
to acetylcholine & & & \\
$R_{\max }$ to SNP (\%) & $99.98 \pm 0.02$ & $99.86 \pm 0.1$ & $99.98 \pm 0.02$ \\
$\mathrm{pD}_{2}$ to SNP & $8.3 \pm 0.1$ & $8.2 \pm 0.1$ & $8.8 \pm 0.6^{\mathrm{a}, \mathrm{b}}$ \\
$\begin{array}{l}\text { Phenylephrine } \\
\text { (\% of KCl) }\end{array}$ & $78.1 \pm 2.5$ & $123.5 \pm 1.6^{\mathrm{a}}$ & $123.6 \pm 3.5^{\mathrm{a}}$ \\
& & &
\end{tabular}

Note. $\mathrm{KCl}=$ potassium chloride.

${ }^{\mathrm{a}} p<.05$ versus control.

${ }^{\mathrm{b}} p<.05$ versus saline. 

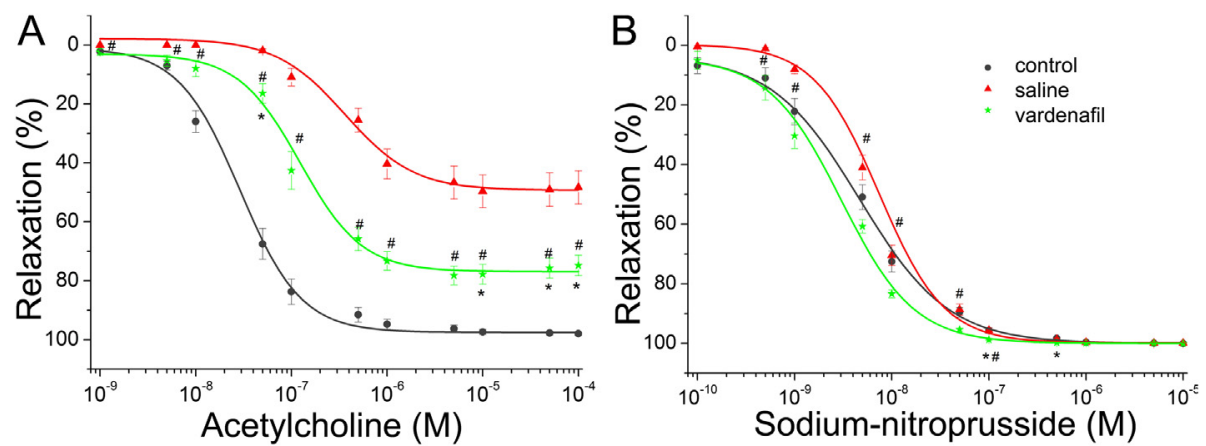

Figure 1. The effect of vardenafil in in vitro ischaemia-reperfusion on vasomotor function of rat aortic rings. (A) Acetylcholine-induced endothelium-dependent vasorelaxation; (B) sodium nitroprusside-induced endothelium-independent vasorelaxation are shown in control, saline, and vardenafil groups. Values represent mean \pm standard error of the mean. Note. ${ }^{*} p<.05$ versus control. ${ }^{\#} p<.05$ versus saline.

in the saline group. Preservation of aortic segments with vardenafil-supplemented saline solution significantly decreased DNA strand breaks (Fig. 2A, C).

We detected significantly lower CGMP immunoreactivity in the saline group than in the control group. A significantly higher score of CGMP staining was observed in the vardenafilsupplemented group than in the saline group (Fig. 2B, D).

\section{Vardenafil regulates aortic gene expression}

Cold ischaemic conservation of aortic rings followed by 30 minutes $\mathrm{HOCl}$ incubation caused statistically relevant upregulation in mRNA expression of the vasoconstrictor endothelin-1 (Fig. 3A) and two apoptotic genes, caspase-3 and bax (Fig. 3B, C), which were significantly decreased by vardenafil. Moreover, the significant down-regulation of
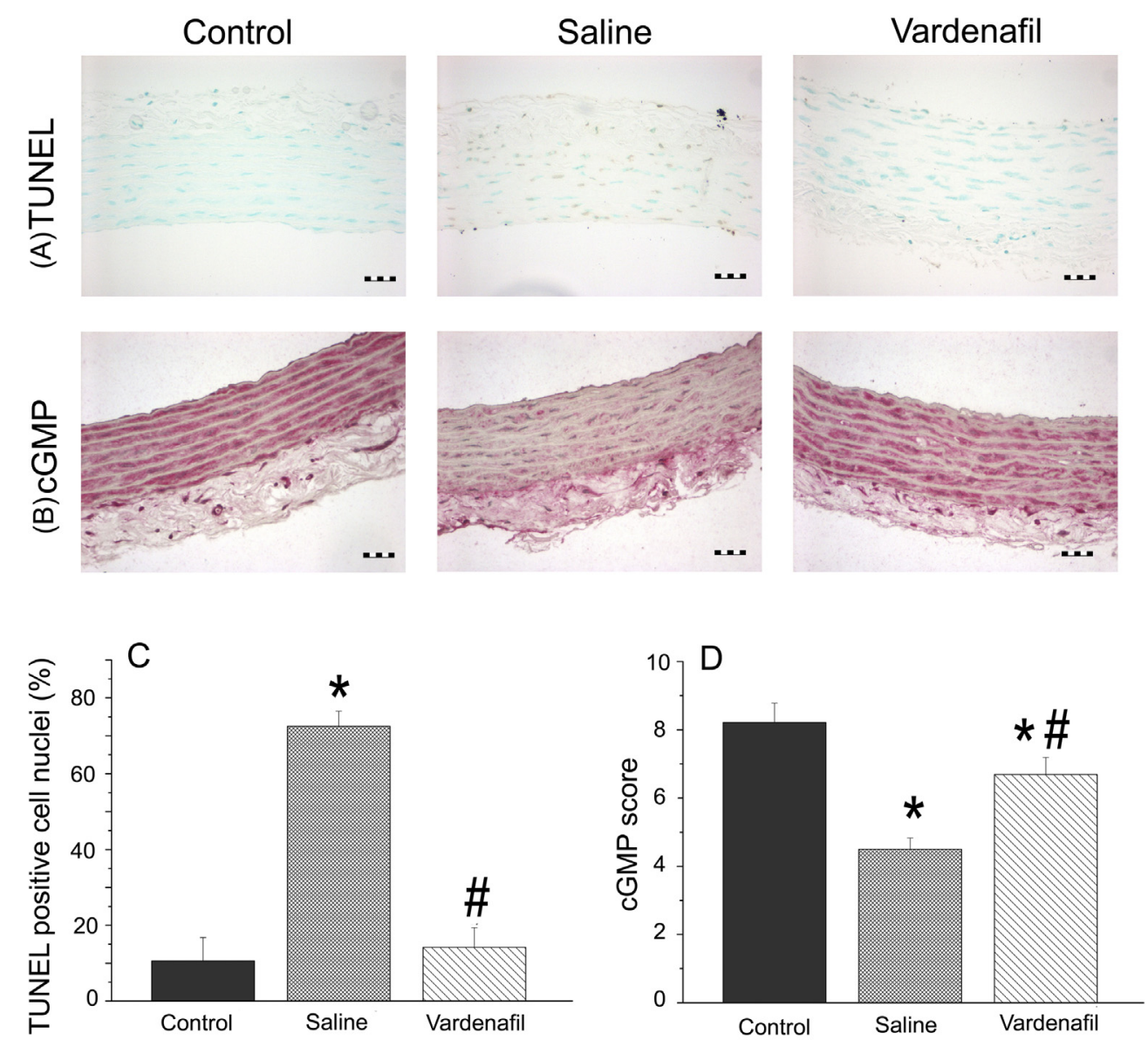

Figure 2. Effects of vardenafil on DNA strand breaks and cyclic guanosine monophosphate (cGMP) levels in aortic rings. (A) Respective photomicrographs of terminal deoxynucleotidyl transferase-mediated dUTP nick-end labelling (TUNEL) reaction (brown staining); (B) immunohistochemical staining for CGMP (red staining) in the vessel wall of control and sodium hypochlorite ( $\mathrm{NaOCl}$ )-exposed, in saline, or in vardenafil $\left(10^{-11} \mathrm{M}\right)$-conserved thoracic aortic rings; (C) scoring of TUNEL staining (in percentage of total cell number); and (D) cGMP immunohistochemistry (magnification $200 \times$, bar $=50 \mu \mathrm{m}$ ). Values represent mean \pm standard error of the mean. Note. ${ }^{*} p<.05$ versus control. ${ }^{\#} p<.05$ versus saline. 
anti-apoptotic Bcl-2 mRNA expression (Fig. 3D) in the saline group was totally antagonised by vardenafil. Vardenafil supplementation did not influence the mRNA level of endothelial nitric oxide synthase (eNOS) and inducible nitric oxide synthase (iNOS) (Fig. 3E, F).

\section{Effect of vardenafil on cleaved caspase-3 level, and Bax and $\mathrm{BCl}-2$ protein expression}

Densitometric analysis of caspase-3 p17 cleavage and Bax bands showed a significant increase in the saline group and was significantly moderated by vardenafil supplement (Fig. 4A, B). Expression of BCl-2 (Fig. 4C) was significantly decreased in saline group and maintained at the level of controls when supplemented with vardenafil.

\section{DISCUSSION}

The major finding of this study is that, with respect to function and structure, long-term storage of rat aortic segments in a vardenafil-supplemented preservation solution provides significantly better protection against I/R injury than the most frequently used saline solution. The beneficial effect of vardenafil was demonstrated by improved vasodilatatory capacity, lower grade DNA strand breaks, maintained cGMP content, and preserved mRNA and protein expression.

To simulate the whole reperfusion process the well established method of reoxygenation and rewarming with additional $\mathrm{HOCl}$ incubation was applied. ${ }^{16,17}$ After prolonged cold storage and high reperfusion levels of ROS, including other less toxic oxygen species, $\mathrm{HOCl}$ is released from activated leucocytes and severely impairs endothelial cells and NO production, and induces apoptosis in endothelial cells and in the vessel wall through oxidative stress. After prolonged cold storage and reperfusion, high levels of ROS severely impair endothelial cells and NO production. Damaged endothelial cells are responsible for impaired vasodilatory graft function and for the developing vasculopathy. ${ }^{20,21}$ Vascular tone (endothelium-dependent vasorelaxation) is particularly important in cardiac, as well as in vascular surgery, as it determines postoperative blood flow and is responsible for the early and late graft thrombosis and stenosis. ${ }^{22}$ Furthermore, the long-term benefit of revascularisation surgery depends heavily on the long-term
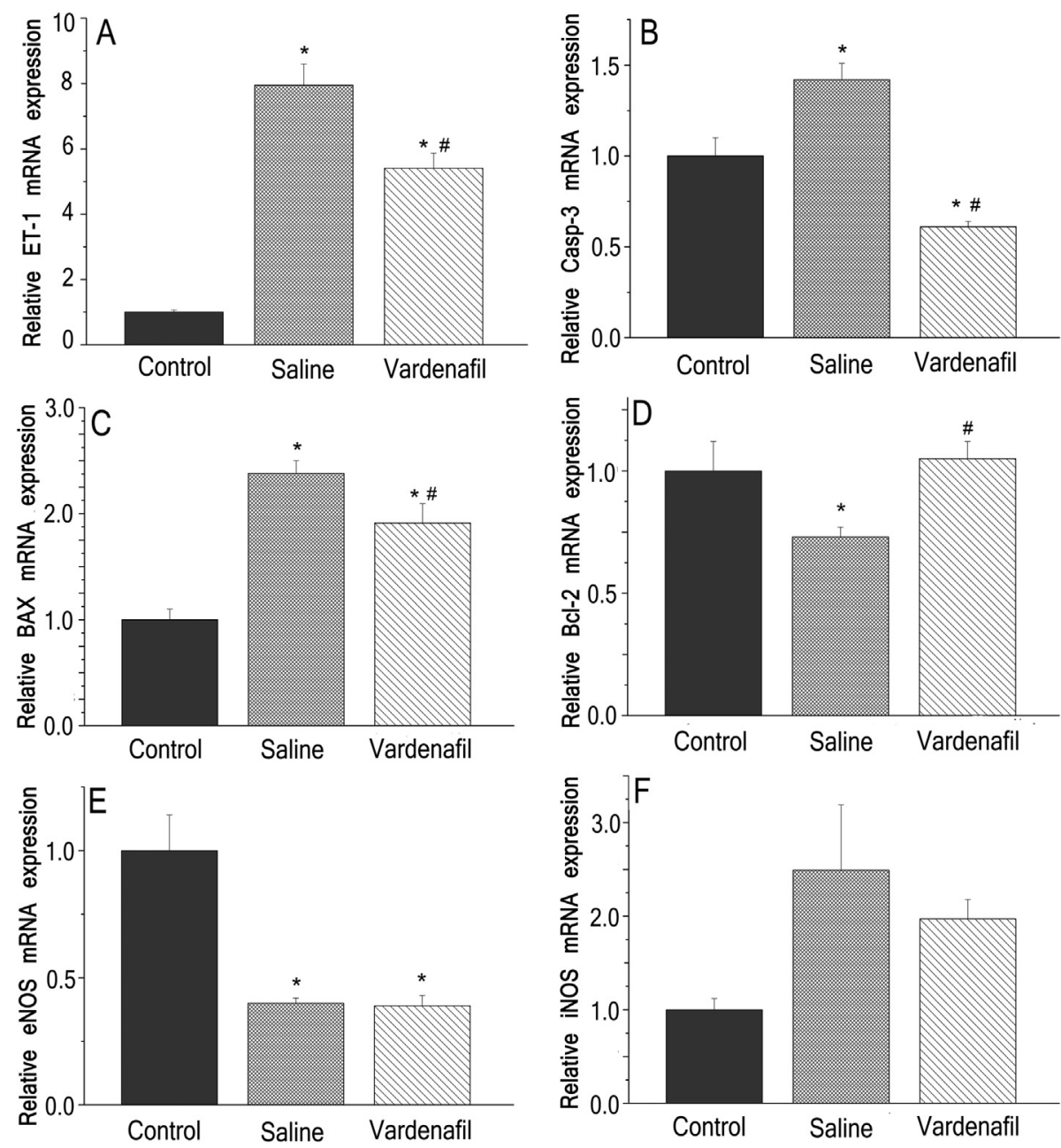

Figure 3. Effects of vardenafil in in vitro ischaemia-reperfusion on the gene expression of (A) endothelin-1 (ET-1), (B) caspase-3 (casp-3), (C) Bax, (D) BCl-2, (E) endothelial nitric oxide synthase (eNOS), and (F) inducible nitric oxide synthase (iNOS) in isolated aortic rings. Controls were given the arbitrary value of 1 . Values represent median \pm quartiles. Note. ${ }^{*} p<.05$ versus control. ${ }^{\#} p<.05$ versus saline. 

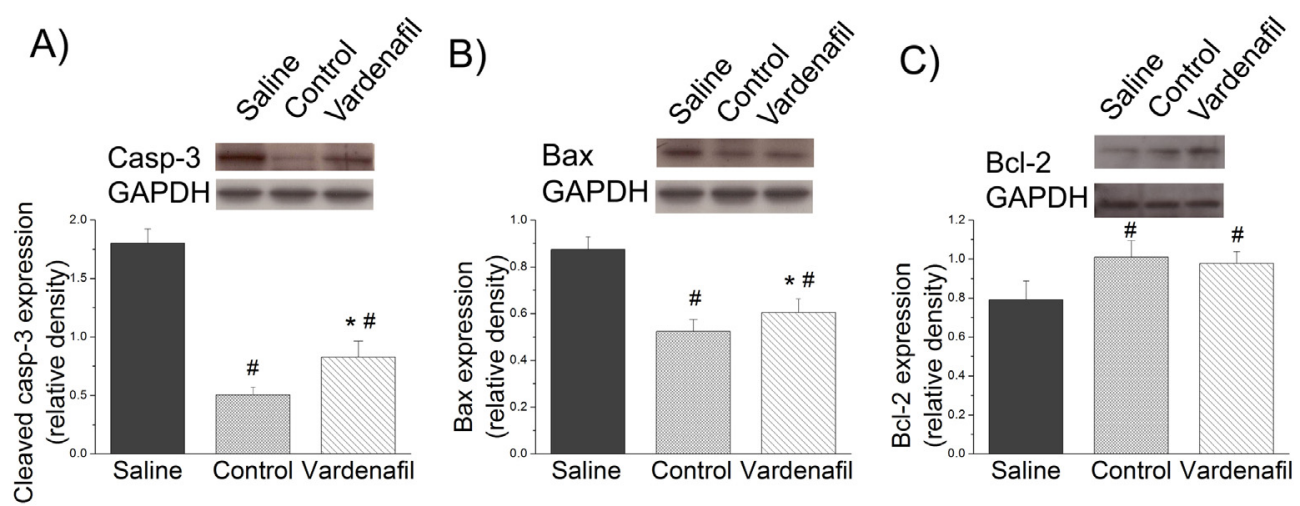

Figure 4. The effects of vardenafil in in vitro ischaemia-reperfusion on protein expression of (A) cleaved caspase-3 (casp-3), (B) Bax, and (C) $\mathrm{Bcl}-2$ in isolated aortic rings ( $n=6$ per group). Values represent mean \pm standard error of the mean. Note. ${ }^{*} p<.05$ versus control. ${ }^{\#} p<.05$ versus saline.

patency of bypass grafts, which is determined by several factors: the progress of heart/vascular disease, the biological properties of the implanted graft, and the degree of $\mathrm{I} / \mathrm{R}$ injury.

Ingemansson et al. ${ }^{23}$ showed weak contractive ability after administration of U-46619, a TXA 2 agonist, after 24 hours of ischaemic storage using University of Wisconsin solution. Another in vitro study demonstrated decreased rat coronary vasodilatory capacity after 8 hours of cold storage in HTK solution. ${ }^{5}$ Different research groups have aimed to develop individual methods to maintain vascular function during storage. ${ }^{17,24}$ In our laboratory, we demonstrated a maintained vascular function with new iron chelators after 24 hours of ischaemia. ${ }^{19}$ In accordance with the literature, in the present study we also found an impaired vasodilatative capacity in aortic rings after prolonged cold storage for 24 hours followed by warm $\mathrm{HOCl}$-induced reperfusion injury by using vitro measurements. ${ }^{16,17}$ The preserved contraction and endothelium-independent relaxation indicated that the impaired vascular response was not a functional deficit of vascular smooth muscle.

Recently, there has been considerable interest in the role of the NO-solube-guanylate-cyclase (sGC)-cGMP-PKG pathway in myocardial and endothelial protection. ${ }^{9,25}$ Previous studies have reported powerful myocardial protection by pharmacological PDE-5 inhibition, ${ }^{1,26}$ by sGC activation, ${ }^{10}$ and by supplementation of an NO-donor, L-Arginin. ${ }^{27}$ Intracellular cGMP-accumulation has been shown to reduce oxidative tissue injury in conditions associated with increased free radical release and oxidative stress. ${ }^{10}$ More recently, the observed cardioprotection with pharmacologically increased cGMP levels has been reported to be mediated by hydrogen sulphide signalling in a PKGdependent fashion. ${ }^{28}$ The PKG-dependent cytoprotective mechanism of vardenafil involves phosphorylation of ERK, induction of $\mathrm{Bcl}-2$, and opening of mitochondrial $\mathrm{K}^{+}$ATP channels. ${ }^{13,29}$ We also demonstrated, in the vardenafiltreated group, up-regulation in both Bcl-2 mRNA and protein expression parallel to a drop in bax and caspase-3 mRNA expressions, and cleaved caspase-3 level, demonstrating inhibition of the apoptotic processes, as also evidenced by the TUNEL staining results. In this study mRNA expression of eNOS and iNOS was significantly decreased in the saline and vardenafil groups. Vardenafil treatment had no effect on these levels. It does not contradict the effect of the PDE-5 inhibitors, as they act through the suppression of cGMP degradation, but have no expected direct effect on NO accumulation. Based on functional improvement we propose that the reduced intracellular cGMP level plays a role in the pathogenesis of vascular dysfunction after 24 hours cold storage, which is also supported by the cGMP immunohistochemistry performed in aortic tissue in this study. Furthermore, severe DNA damage could also be effectively prevented by vardenafil, as evidenced by our TUNEL labelling.

To the best of our knowledge, it has not been previously tested whether the selective inhibition of PDE- 5 would also be capable of preventing I/R injury after long-term storage.

In conclusion, we report, for the first time, that the PDE-5 inhibitor vardenafil added to a preservation solution restored impaired endothelial function of aortic rings after long-term cold storage followed by $\mathrm{HOCl}$-induced warm reperfusion. This work supports the view that PDE5 inhibition might be advantageous in the treatment of endothelial dysfunction during long-term storage.

\section{Study limitations}

The PDE-5 inhibitor vardenafil may have therapeutic potential to lower oxidative stress with the aim of improving clinical outcome of $I / R$ injury in blood vessels after transplantation. However, in our study, aortic rings were harvested ex vivo to examine vascular reactivity without the involvement of non-aortic tissue, with lack of blood flow and absence of leucocyte activation. Therefore, confirmation of these observations in vivo is essential. Also, 24 hours of cold ischaemic storage is validated as part of the wellestablished in vitro vascular model of $I / R$, but is not common in clinical practice. Moreover, additional studies would be interesting to investigate the effect of vardenafil on venous grafts.

\section{CONFLICT OF INTEREST}

None. 


\section{FUNDING}

None.

\section{APPENDIX A. SUPPLEMENTARY MATERIAL}

Supplementary data related to this article can be found online at http://dx.doi.org/10.1016/j.ejvs.2013.05.006.

\section{REFERENCES}

1 Szabo G, Radovits T, Veres G, Krieger N, Loganathan S, Sandner $\mathrm{P}$, et al. Vardenafil protects against myocardial and endothelial injuries after cardiopulmonary bypass. Eur J Cardiothorac Surg 2009;36:657-64.

2 Maathuis MH, Leuvenink HG, Ploeg RJ. Perspectives in organ preservation. Transplantation 2007;83:1289-98.

3 Schaeffer U, Tanner B, Strohschneider T, Stadtmuller A, Hannekum A. Damage to arterial and venous endothelial cells in bypass grafts induced by several solutions used in bypass surgery. Thorac Cardiovasc Surg 1997;45:168-71.

4 Wilbring $M$, Tugtekin SM, Zatschler B, Ebner A, Reichenspurner $\mathrm{H}$, Matschke $\mathrm{K}$, et al. Even short-time storage in physiological saline solution impairs endothelial vascular function of saphenous vein grafts. Eur J Cardiothorac Surg 2011;40:811-5.

5 Schroder C, Heintz A, Pexa A, Rauen U, Deussen A. Preclinical evaluation of coronary vascular function after cardioplegia with HTK and different antioxidant additives. Eur J Cardiothorac Surg 2007;31:821-6.

6 Zweier JL, Talukder MA. The role of oxidants and free radicals in reperfusion injury. Cardiovasc Res 2006;70:181-90.

7 Korkmaz S, Radovits T, Barnucz E, Neugebauer P, Arif R, Hirschberg K, et al. Dose-dependent effects of a selective phosphodiesterase-5-inhibitor on endothelial dysfunction induced by peroxynitrite in rat aorta. Eur J Pharmacol 2009;615:155-62.

8 Boyle Jr EM, Pohlman TH, Cornejo CJ, Verrier ED. Endothelial cell injury in cardiovascular surgery: ischemia-reperfusion. Ann Thorac Surg 1996;62:1868-75.

9 Kukreja RC, Ockaili R, Salloum F, Yin C, Hawkins J, Das A, et al. Cardioprotection with phosphodiesterase-5 inhibition - a novel preconditioning strategy. J Mol Cell Cardiol 2004;36: 165-73.

10 Korkmaz S, Radovits T, Barnucz E, Hirschberg K, Neugebauer P, Loganathan $S$, et al. Pharmacological activation of soluble guanylate cyclase protects the heart against ischemic injury. Circulation 2009;120:677-86.

11 Abdollahi M, Fooladian F, Emami B, Zafari K, BahreiniMoghadam A. Protection by sildenafil and theophylline of lead acetate-induced oxidative stress in rat submandibular gland and saliva. Hum Exp Toxicol 2003;22:587-92.

12 Dias-Junior CA, Souza-Costa DC, Zerbini T, da Rocha JB, Gerlach RF, Tanus-Santos JE. The effect of sildenafil on pulmonary embolism-induced oxidative stress and pulmonary hypertension. Anesth Analg 2005;101:115-20.

13 Salloum FN, Ockaili RA, Wittkamp M, Marwaha VR, Kukreja RC. Vardenafil: a novel type 5 phosphodiesterase inhibitor reduces myocardial infarct size following ischemia/reperfusion injury via opening of mitochondrial K(ATP) channels in rabbits. $J$ Mol Cell Cardiol 2006;40:405-11.
14 Mazo E, Gamidov S, Iremashvili V. The effect of vardenafil on endothelial function of brachial and cavernous arteries. Int J Impot Res 2006;18:464-9.

15 Gori T, Sicuro S, Dragoni S, Donati G, Forconi S, Parker JD. Sildenafil prevents endothelial dysfunction induced by ischemia and reperfusion via opening of adenosine triphosphate-sensitive potassium channels: a human in vivo study. Circulation 2005;111:742-6.

16 Radovits T, Lin LN, Zotkina J, Gero D, Szabó C, Karck M, et al. Poly(ADP-ribose) polymerase inhibition improves endothelial dysfunction induced by reactive hydrogen peroxide in vitro. Eur J Pharmacol 2007;564:158-66.

17 Barnucz E, Veres G, Hegedus P, Klein S, Zoller R, Radovits T, et al. Prolyl-hydroxylase inhibition preserves endothelial cell function in a rat model of vascular ischemia reperfusion injury. J Pharmacol Exp Ther 2013;345:25-31.

18 Hirschberg K, Radovits T, Loganathan S, Entz L, Beller CJ, Gross ML, et al. Selective phosphodiesterase-5 inhibition reduces neointimal hyperplasia in rat carotid arteries after surgical endarterectomy. J Thorac Cardiovasc Surg 2009;137: 1508-14.

19 Radovits T, Lin LN, Zotkina J, Koch A, Rauen U, Köhler G, et al. Endothelial dysfunction after long-term cold storage in HTK organ preservation solutions: effects of iron chelators and $\mathrm{N}$ alpha-acetyl-L-histidine. J Heart Lung Transplant 2008;27:20816.

20 Chu Y, Wu YC, Chou YC, Chueh HY, Liu HP, Chu JJ, et al. Endothelium-dependent relaxation of canine pulmonary artery after prolonged lung graft preservation in University of Wisconsin solution: role of L-arginine supplementation. J Heart Lung Transplant 2004;23:592-8.

21 Zhang RZ, Yang Q, Yim AP, He GW. Alteration of cellular electrophysiologic properties in porcine pulmonary microcirculation after preservation with University of Wisconsin and EuroCollins solutions. Ann Thorac Surg 2004;77:1944-50.

$22 \mathrm{He} \mathrm{GW}$. Endothelial function related to vascular tone in cardiac surgery. Heart Lung Circ 2005;14:13-8.

23 Ingemansson R, Sjoberg T, Massa G, Steen S. Long-term preservation of vascular endothelium and smooth muscle. Ann Thorac Surg 1995;59:1177-81.

24 Zatschler B, Dieterich P, Muller B, Kasper M, Rauen U, Deussen A. Improved vessel preservation after 4 days of cold storage: experimental study in rat arteries. J Vasc Surg 2009;50:397-406.

25 Kukreja RC, Salloum FN, Das A. Cyclic guanosine monophosphate signaling and phosphodiesterase-5 inhibitors in cardioprotection. J Am Coll Cardiol 2012;29:1921-7.

26 Radovits T, Bomicke T, Kokeny G, Arif R, Loganathan S, Kecsan K, et al. The phosphodiesterase- 5 inhibitor vardenafil improves cardiovascular dysfunction in experimental diabetes mellitus. Br J Pharmacol 2009;156:909-19.

27 Soos P, Andrasi T, Buhmann V, Kohl B, Vahl C, Hagl S, et al. Myocardial protection after systemic application of L-arginine during reperfusion. J Cardiovasc Pharmacol 2004;43:782-8.

28 Salloum FN, Das A, Samidurai A, Hoke NN, Chau VQ, Ockaili RA, et al. Cinaciguat, a novel activator of soluble guanylate cyclase, protects against ischemia/reperfusion injury: role of hydrogen sulfide. Am J Physiol Heart Circ Physiol 2012;302:H1347-54.

29 Das A, Salloum FN, Xi L, Rao YJ, Kukreja RC. ERK phosphorylation mediates sildenafil-induced myocardial protection against ischemia-reperfusion injury in mice. Am J Physiol Heart Circ Physiol 2009;296:H1236-43. 